\title{
A newly discovered role of telomeres in an ancient organism
}

Bibo Li

Cleveland State University; Center for Gene Regulation in Health and Disease; Department of Biological, Geological and Environmental Sciences; Cleveland, OH USA

Key words: antigenic variation, telomere position effect, RAP1, Trypanosoma brucei, variant surface glycoprotein, gene silencing, monoallelic expression, microbial pathogen virulence

Submitted: 01/29/10

Revised: 03/02/10

Accepted: 03/03/10

Previously published online: www.landesbioscience.com/journals/ nucleus/article/11742

*Correspondence to:

Bibo Li; Email: b.li37@csuohio.edu

ExtraView: Yang $X$, Figueiredo LM, Espinal A, Okubo E, Li B. RAP1 is essential for silencing telomeric variant surface glycoprotein genes in Trypanosoma brucei. Cell 2009; 137:99-109;

PMID: 19345190; DOI: 10.1016/j.cell.2009.01.037.
Typanosoma brucei expresses Variant Surface Glycoprotein (VSG) genes in a strictly monoallelic fashion in its mammalian hosts, and the regulation of this important virulence mechanism has been the research focus for decades. Telomere position effect (TPE), an epigenetic phenomenon, has been proposed to play a critical role in VSG regulation, yet no telomeric protein was identified whose disruption led to VSG derepression. We recently identified tbRAP1 as an intrinsic component of the T. brucei telomere complex and a major regulator for silencing VSG expression sites (ESs). Knockdown of tbRAP1 led to derepression of all ES-linked VSGs but not VSGs located elsewhere, and resulted in stronger derepression of telomere-proximal genes than telomere-distal genes. This tapered silencing pattern further argues that telomere integrity plays a key role in tbRAP1-dependent silencing and for the first time provides direct evidence indicating that telomeres are important for VSG expression regulation. Whether chromatin remodeling is important for tbRAP1-mediated silencing as in classical TPE will also be discussed.

\section{Monoallelic Expression of VSG and Antigenic Variation in Trypanosoma brucei}

Human African Trypanosomiasis (HAT) is a tropical disease that threatens more than 60 million people in sub-Sahara Africa. According to $\mathrm{WHO}$, between 300,000 and 500,000 people are currently infected. Without treatment, HAT is inevitably fatal. The culprit for HAT is a protozoan parasite called Trypanosoma brucei, and the disease is transmitted through bites of infected tsetse flies (Glossina species). T. brucei also causes Nagana in cattle, which has profoundly affected the settlement and economic development of a major part of Africa.

Once an infection is established, it is very hard to eliminate T. brucei from its mammalian host. This is mainly because bloodstream form (BF) T. brucei can evade the mammalian host immune attack by regularly switching its surface coat, a phenomenon termed antigenic variation. ${ }^{1}$ To maximize the switching efficiency and effectiveness, at any time only a single type of variant surface glycoprotein (VSG) is expressed. ${ }^{2}$ Hence both monoallelic VSG expression and VSG switching are critical for persistent T. brucei infection.

Although there are more than 1,000 VSG genes and pseudogenes in T. brucei genome, ${ }^{3}$ VSGs are exclusively expressed from subtelomeric expression sites (ESs), ${ }^{4}$ which are polycistronically transcribed by RNA polymerase I. ${ }^{5}$ Each ES contains multiple genes, and VSG is the last one in the unit, usually $40-60 \mathrm{~kb}$ downstream of the promoter but within $1.6 \mathrm{~kb}$ of the telomere repeats. ${ }^{6}$ There are $15 \mathrm{ESs}$ in the T. brucei 427 strain, 14 of which have distinctive VSGs, but otherwise their sequences are $90 \%$ identical. ${ }^{6}$ However, only one ES promoter is fully active at any time, resulting in a single type of VSG being expressed. This allelic exclusion ensures that the previously active VSG is turned off after a switching event so that the cell is no longer recognizable by the host immune system.

Although the monoallelic expression of VSG has been studied for more than 30 years, how it is regulated was poorly 
understood. Only recently did we begin to reveal its underlying mechanisms. Here I will refer to several recent key findings, focusing on our studies on tbRAP1's functions, and discuss our current model of VSG expression regulation.

\section{Telomeres are Important for Monoallelic VSG Expression}

Because VSGs are exclusively expressed from subtelomeric loci, ${ }^{4,6}$ it has long been hypothesized that telomeres may play an important role in VSG expression regulation. ${ }^{7}$ This was a very attractive hypothesis particularly after the discovery that telomeres often form a heterochromatic structure that suppresses the transcription of genes located nearby. This Telomere Position Effect (TPE) was first identified in budding yeast ${ }^{8}$ and subsequently demonstrated in several other organisms including fission yeast, T. brucei, and mammalian cells. ${ }^{9-11}$ Specifically in T. brucei, when an rDNA promoterdriven reporter gene was inserted at a subtelomeric locus, either inside a silent ES or at a non-ES subtelomere, it was more strongly silenced when it was at telomereproximal loci than at telomere-distal loci. ${ }^{10,12}$ However, for decades, there was no direct evidence to support the involvement of telomeres in VSG expression regulation. Several observations even suggested the opposite. Deletion of telomerase, the enzyme that synthesizes telomere DNA de novo, or $\mathrm{Ku}$, whose yeast homolog plays an important role in TPE, only led to progressive telomere shortening but did not affect TPE or VSG silencing. ${ }^{13-15}$ Deletion of T. brucei Sir2, which, like its yeast and mammalian homologs, is an $\mathrm{NAD}^{+}$-dependent histone deacetylase, ${ }^{16}$ only abolished silencing of subtelomeric rDNA promoter-driven reporter genes but not VSGs. ${ }^{16,17}$ In addition, a truncated telomere did not lead to expression of the adjacent silent ES-linked VSG. ${ }^{18}$ Even depletion of the duplex telomere DNA binding protein, tbTRF, did not seem to affect VSG expression. ${ }^{19}$ Therefore, it was a great breakthrough when $T$. brucei RAP1, an intrinsic component of the telomere complex, was shown to play an essential role in silencing ES-linked VSGs. ${ }^{20}$
The most striking phenotype observed in cells depleted of tbRAP1 is that all silent ES-linked VSGs were derepressed tens to hundreds of fold (variations were observed in different cell lines and for different VSGs). ${ }^{20}$ VSG proteins were synthesized from the derepressed genes and transported to the cell surface. Furthermore, more than one newly synthesized VSG proteins in addition to the originally active one were observed on the surface of individual cells, indicating that multiple VSGs were expressed in the same cells at the same time. Therefore, tbRAP1 plays an essential role in silencing ES-linked VSGs.

We found that tbRAP1 only affected transcription of ES-linked subtelomeric VSG genes but not promoter-less VSGs. In addition, tbRAP1 did not affect transcription of any chromosome internally located control genes including a ribosomal protein gene, a glycolytic protein gene, rDNA, and the telomerase protein component gene, indicating that tbRAP1 is not a general transcription repressor. Furthermore, using quantitative RT-PCR, we found that tbRAP1-mediated silencing effect was stronger for telomere-proximal genes than telomere-distal genes, arguing that tbRAP1-medated silencing depends on the telomere structure and that telomeres indeed play an important role in VSG expression control.

\section{Involvement of Chromatin Remodeling in VSG Silencing}

TbRAP1-mediated silencing is similar to TPE but also has many unique features. TPE is best studied in budding yeast, where $S$. cerevisiae RAP1 is the predominant duplex telomere DNA binding factor $^{21}$ and plays an essential role in both establishing and maintenance of TPE. ${ }^{22}$ It has been shown that $S$. cerevisiae RAP1 and $\mathrm{Ku}$ bind telomere DNA and recruit Sir2/3/4 to the telomere vicinity. ${ }^{23}$ This initiates/nucleates the heterochromatic structure at the telomere. The deacetylation activity of Sir2 creates a high-affinity site for the Sir complex and help the complex and silencing to spread towards chromosome internal loci. ${ }^{24-26}$ Therefore, the hypoacetylated chromatin structure is essential for telomeric silencing in yeast, which depends on the telomere localization of RAP1. The degree of silencing is inversely proportional to the distance between the reporter gene and the telomeric tract, ${ }^{22,27}$ and the silencing status is reversible, reflecting a competition between the transcription and silencing machineries. ${ }^{8}$ Most studies in yeast used RNA Pol II transcribed auxotroph marker targeted to subtelomeric region as an indicator for silencing. At these marked and truncated chromosome ends, silencing strength tapers continuously from the telomere to a few kilo-bases upstream. ${ }^{8}$ Telomeric silencing at different native chromosome ends is of various strengths, and the peak silencing can be detected at places not immediately adjacent to the telomere tract. $^{28}$

Histone hypoacetylation is also important for TPE in human cells, ${ }^{11,29,30}$ as silencing can be disrupted by treatment with the deacetylase inhibitor TSA. In addition, both telomeric and subtelomeric chromatin are enriched in trimethylated $\mathrm{H} 3 \mathrm{~K} 9$ and $\mathrm{H} 4 \mathrm{~K} 20$, typical histone modifications found in heterochromatic structure, ${ }^{31,32}$ indicating that more sophisticated chromatin modification mechanisms are involved.

Little is known about the mechanisms underlying tbRAP1-mediated silencing. However, it is position-dependent, ${ }^{20}$ and the chromatin structure seems to be important (see below). TbRAP1-mediated silencing is unusual in that it affects RNA Pol I transcription and spreads more than $50 \mathrm{~kb}$ upstream of the telomere. It is possible that tbRAP1-mediated silencing may also suppress subtelomeric RNA Pol II transcription but this has not been tested. So far, none of the RNA Pol II transcribed genes has been identified at any subtelomeric regions, and RNA Pol II specific promoters are not very well characterized in T. brucei. In addition, although T. brucei Sir2 homolog appears to be important for telomeric silencing of the rDNA promoterdriven reporter genes, it is not required for VSG silencing. ${ }^{17}$ Therefore, tbRAP1mediated silencing does not appear to depend on histone deacetylation.

Several recent reports have shown that chromatin remodeling plays an important role in VSG expression regulation. Specifically, depletion of tbISWI, 
a chromatin remodeling homolog, led to a 30- to 60-fold increase in ES promoter proximal gene transcription but did not abolish VSG silencing, ${ }^{33}$ while deletion of tbDotlb, a histone methyltransferase, led to a 10-fold increase in silent ES transcription, including ES-linked VSGs. ${ }^{34}$ In addition, silent ESs have a normal chromatin structure with regularly spaced nucleosomes, while the active ES only has a small number of nucleosomes that are irregularly positioned, suggesting that nucleosome deposition may be important for VSG control. ${ }^{35,36}$ Interestingly, we found that depletion of tbRAP1 led to decreased amount of nucleosomes at the derepressed ESs (Pandya U, Li B, unpublished data), suggesting that tbRAP1-mediated silencing may act through regulation of chromatin remodeling, which appears to be a common player for ES silencing and TPE in various organisms, although the detailed mechanisms may different.

\section{Silencers of VSG ESs}

Our and others' studies have identified three silencing factors at ESs. TbISW1 suppresses ES promoter activity for 30-60 fold, ${ }^{33}$ tbDotlb decreases the whole ES transcription for 10 -fold, ${ }^{34}$ while tbRAP1 causes up to hundreds of fold of reduction in VSG expression and up to 30 -fold in transcription of genes immediately downstream of the ES promoter. ${ }^{20}$ It is worth noting that phenotypes observed in cells depleted of tbISW1 or tbRAP1 may not reflect the full extent of these proteins' effects, as RNAi-mediated knockdown may not remove the targeted proteins as completely as double-allele deletion. Neither tbISW1 nor tbDot1b affects ES transcription the same way as tbRAP1, for their depletion or deletion phenotypes are distinctive from tbRAP1 depletion phenotypes. ${ }^{20,33,34}$ However, it is necessary to examine phenotypes in cells depleted of a various combination of any two or all of the three proteins to determine whether they function in the same genetic pathway.

Should all these ES repressors work independently, their effects would be additive and removal of all three factors is expected to lead to thousands of fold of increase in VSG transcription. This may result in transcription of derepressed ESs at a level similar to the fully active ES, because quantitative RT-PCR analyses have shown that the active ES transcribes its VSG gene at a level upto 10,000-fold higher than silent ESs. ${ }^{20}$ It will be interesting to see whether the difference between the active and silent ES transcription can be accounted for by the combined action of all three ES repressors, as this will also provide clues to whether additional mechanisms are necessary for ES transcription regulation.

We anticipate that regulation of ES expression is more complicated than involving only three repressors. It has been shown that only the active ES is colocalized with ample RNA Pol I at the extranucleolar ES Body (ESB). ${ }^{37}$ Therefore, it is likely that transcription of ES is ultimately limited by RNA pol I availability.

\section{Mechanisms of Monoallelic VSG Expression Regulation}

Recent studies helped us to better understand how T. brucei cells silence ES-linked VSGs. However, it is still unclear how one particular ES is selected for transcription. It has been shown that only the active ES is colocalized with ESB, which appears to be an extranucleolar entity that is enriched with RNA Pol I. ${ }^{37}$ Therefore, it appears that only association with ESB will allow the ES to get access to sufficient RNA Pol I to support the extremely high level of VSG transcription $\left(\sim 10^{7}\right.$ molecules of VSG are made per cell, which accounts for one tenth of the total cellular proteins). It is interesting that in cells depleted of tbRAP1, we observed multiple extranucleolar RNA Pol I foci with various staining intensity, ${ }^{20}$ reflecting that multiple ESs are transcribed simultaneously. Because derepressed ESs are only transcribed at a moderate level, this observation further suggested that an ES has to associate with ESB to be fully transcribed. Therefore, limiting RNA Pol I accessibility may be an additional mechanism that prevents transcription of multiple ESs at the fully active level simultaneously. In support of this, two forced fully active ESs have to switch back and force rapidly and locate next to each other in the nucleus, suggesting that the two ESs are competing with a limiting factor that is essential for full level of transcription. ${ }^{38}$
Considering all facts accumulated so far, our current working model hypothesizes that ESs probably assume by default an active or semi-active state in BF cells, so that they can be rapidly switched to be fully active. However, this "ready to go" state needs to be "checked" or suppressed for all but one ESs, and tbISW1, tbDOT1b, tbRAP1, and possibly other silencing machineries are necessary for this task. As an additional check, the accessibility of RNA Pol I machinery may also be limited, which could be mediated by an unknown mechanism that actively recruits one and only one ES to ESB for full transcription.

\section{Telomeres in Regulation of Microbial Pathogen Virulence}

Although TPE has been identified for nearly two decades, most of the earlier works were done with reporter genes and it was questioned whether TPE has any intrinsic biological functions. However, studies in recent years have indicated that TPE is important for virulence regulation in some microbial pathogens. In Candida glabrata, the causal agent of opportunistic urogenital tract and bloodstream infections in immunocompromised individuals, epithelial adhesin is one of the virulence factors. Adhesins are encoded by the subtelomere-localized $E P A$ gene family, and silencing of EPA genes is dependent on TPE. ${ }^{39,40}$ In addition, Plasmodium falciparum, the causal pathogen for malaria, expresses variant surface antigen encoded by the var genes in a monoallelic fashion at the trophozoite stage. Many var genes are located at subtelomeric regions, and their silencing is dependent on Sir2-mediated TPE. ${ }^{41,42}$ Furthermore, our recent findings showed that $T$. brucei silences its surface antigen genes through a similar telomere-based silencing mechanism, ${ }^{20}$ further supporting the hypothesis that telomeres are important for regulation of the expression of surface antigen genes in quite a few microbial pathogens. Further studies on telomere functions in T. brucei and other microbial pathogens will help to advance our knowledge in this area.

Telomeres are essential for maintaining linear chromosome stability and eukaryotic 
cell proliferation potential. Our recent studies in the kinetoplastid Trypanosoma brucei demonstrated that telomeres are also important for pathogen virulence in an ancient organism. Apparently, although telomeres are located at the ends of linear chromosomes, they are a central player for many essential cellular functions.

\section{References}

1. Barry JD, McCulloch R. Antigenic variation in trypanosomes: enhanced phenotypic variation in a eukaryotic parasite. Adv Parasitol 2001; 49:1-70.

2. Cross GAM. Identification, purification and properties of clone-specific glycoprotein antigens constituting the surface coat of Trypanosoma brucei. Parasitology 1975; 71:393-417.

3. Berriman M, Ghedin E, Hertz-Fowler C, Blandin G, Renauld H, Bartholomeu DC, et al. The genome of the African trypanosome Trypanosoma brucei. Science 2005; 309:416-22.

4. de Lange T, Borst P. Genomic environment of the expression-linked extra copies of genes for surface antigens of Trypanosoma brucei resembles the end of a chromosome. Nature 1982; 299:451-3.

5. Gunzl A, Bruderer T, Laufer G, Schimanski B, Tu LC, Chung HM, et al. RNA polymerase I transcribes procyclin genes and variant surface glycoprotein gene expression sites in Trypanosoma brucei. Eukaryot Cell 2003; 2:542-51.

6. Hertz-Fowler C, Figueiredo LM, Quail MA, Becker M, Jackson A, Bason N, et al. Telomeric expression sites are highly conserved in Trypanosoma brucei. PLoS ONE 2008; 3:3527.

7. Dreesen $\mathrm{O}, \mathrm{Li}$ B, Cross GAM. Telomere structure and function in trypanosomes: a proposal. Nat Rev Microbiol 2007; 5:70-5.

8. Gottschling DE, Aparicio OM, Billington BL, Zakian VA. Position effect at S. cerevisiae telomeres: reversible repression of pol II transcription. Cell 1990; 63:751-62.

9. Nimmo ER, Cranston G, Allshire RC. Telomereassociated chromosome breakage in fission yeast results in variegated expression of adjacent genes. EMBO J 1994; 13:3801-11.

10. Horn D, Cross GAM. A developmentally regulated position effect at a telomeric locus in Trypanosoma brucei. Cell 1995; 83:555-61.

11. Baur JA, Zou Y, Shay JW, Wright WE. Telomere position effect in human cells. Science 2001; 292:2075207.

12. Glover L, Horn D. Repression of polymerase I-mediated gene expression at Trypanosoma brucei telomeres. EMBO Rep 2006; 7:93-9.

13. Dreesen O, Li B, Cross GAM. Telomere structure and shortening in telomerase-deficient Trypanosoma brucei. Nuc Acids Res 2005; 33:4536-43.
14. Janzen CJ, Lander F, Dreesen O, Cross GAM. Telomere length regulation and transcriptional silencing in KU80-deficient Trypanosoma brucei. Nuc Acids Res 2004; 32:6575-84.

15. Conway C, McCulloch R, Ginger ML, Robinson NP, Browitt A, Barry JD. Ku is important for telomere maintenance, but not for differential expression of telomeric VSG genes, in African trypanosomes. J Biol Chem 2002; 277:21269-77.

16. Garcia-Salcedo JA, Gijon P, Nolan DP, Tebabi P, Pays E. A chromosomal SIR2 homologue with both histone NAD-dependent ADP-ribosyltransferase and deacetylase activities is involved in DNA repair in Trypanosoma brucei. EMBO J 2003; 22:5851-62.

17. Alsford S, Kawahara T, Isamah C, Horn D. A sirtuin in the African trypanosome is involved in both DNA repair and telomeric gene silencing but is not required for antigenic variation. Mol Microbiol 2007; 63:72436.

18. Glover L, Alsford S, Beattie C, Horn D. Deletion of a trypanosome telomere leads to loss of silencing and progressive loss of terminal DNA in the absence of cell cycle arrest. Nuc Acids Res 2007; 35:872-80.

19. Li B, Espinal A, Cross GAM. Trypanosome telomeres are protected by a homologue of mammalian TRF2 Mol Cell Biol 2005; 25:5011-21.

20. Yang X, Figueiredo LM, Espinal A, Okubo E, Li B. RAP1 is essential for silencing telomeric variant surface glycoprotein genes in Trypanosoma brucei. Cell 2009; 137:99-109.

21. Konig P, Giraldo R, Chapman L, Rhodes D. The crystal structure of the DNA-binding domain of yeast RAP1 in complex with telomeric DNA. Cell 1996; 85:125-36.

22. Kyrion G, Liu K, Liu C, Lustig AJ. RAP1 and telomere structure regulate telomere position effects in Saccharomyces cerevisiae. Genes Dev 1993; 7:1146-59.

23. Luo K, Vega-Palas MA, Grunstein M. Rap1-Sir4 binding independent of other Sir, $\mathrm{yKu}$, or histone interactions initiates the assembly of telomeric heterochromatin in yeast. Genes Dev 2002; 16:1528-39.

24. Moretti P, Freeman K, Coodly L, Shore D. Evidence that a complex of SIR proteins interacts with the silencer and telomere-binding protein RAP1. Genes Dev 1994; 8:2257-69.

25. Mishra K, Shore D. Yeast Ku protein plays a direct role in telomeric silencing and counteracts inhibition by Rif proteins. Curr Biol 1999; 9:1123-6.

26. Blander G, Guarente L. The Sir2 family of protein deacetylases. Annu Rev Biochem 2004; 73:417-35.

27. Renauld H, Aparicio OM, Zierath PD, Billington BL, Chhablani SK, Gottschling DE. Silent domains are assembled continuously from the telomere and are defined by promoter distance and strength, and by SIR3 dosage. Genes Dev 1993; 7:1133-45.

28. Pryde FE, Louis EJ. Limitations of silencing at native yeast telomeres. EMBO J 1999; 18:2538-50.
29. Pedram M, Sprung CN, Gao Q, Lo AW, Reynolds GE, Murnane JP. Telomere position effect and silencing of transgenes near telomeres in the mouse. Mol Cell Biol 2006; 26:1865-78.

30. Koering CE, Pollice A, Zibella MP, Bauwens S, Puisieux A, Brunori M, et al. Human telomeric position effect is determined by chromosomal contex and telomeric chromatin integrity. EMBO Rep 2002; 3:1055-61.

31. Schoeftner S, Blasco MA. A 'higher order' of telomere regulation: telomere heterochromatin and telomeric RNAs. EMBO J 2009; 28:2323-36.

32. Blasco MA. The epigenetic regulation of mammalian telomeres. Nat Rev Genet 2007; 8:299-309.

33. Hughes K, Wand M, Foulston L, Young R, Harley $\mathrm{K}$, Terry $\mathrm{S}$, et al. A novel ISWI is involved in VSG expression site downregulation in African trypanosomes. EMBO J 2007; 26:2400-10.

34. Figueiredo LM, Janzen CJ, Cross GAM. A histone methyltransferase modulates antigenic variation in African trypanosomes. PLoS Biol 2008; 6:161.

35. Stanne TM, Rudenko G. Active VSG expression sites in Trypanosoma brucei are depleted of nucleosomes. Eukaryot Cell 2010; 9:136-47.

36. Figueiredo LM, Cross GAM. Nucleosomes are depleted at the VSG expression site transcribed by RNA polymerase I in African trypanosomes. Eukaryot Cell 2010; 9:148-54.

37. Navarro M, Gull K. A pol I transcriptional body associated with VSG mono-allelic expression in Trypanosoma brucei. Nature 2001; 414:759-63.

38. Chaves I, Rudenko G, Dirks-Mulder A, Cross M, Borst P. Control of variant surface glycoprotein gene-expression sites in Trypanosoma brucei. EMBO J 1999; 18:4846-55.

39. De Las Penas A, Pan SJ, Castano I, Alder J, Cregg R, Cormack BP. Virulence-related surface glycoproteins in the yeast pathogen Candida glabrata are encoded in subtelomeric clusters and subject to RAP1- and SIR-dependent transcriptional silencing. Genes Dev 2003; 17:2245-58.

40. Castano I, Pan SJ, Zupancic M, Hennequin C, Dujon B, Cormack BP. Telomere length control and transcriptional regulation of subtelomeric adhesins in Candida glabrata. Mol Microbiol 2005; 55:1246-58.

41. Freitas-Junior LH, Hernandez-Rivas R, Ralph SA, Montiel-Condado D, Ruvalcaba-Salazar OK, RojasMeza AP, et al. Telomeric heterochromatin propagation and histone acetylation control mutually exclusive expression of antigenic variation genes in malaria parasites. Cell 2005; 121:25-36

42. Duraisingh MT, Voss TS, Marty AJ, Duffy MF, Good RT, Thompson JK, et al. Heterochromatin silencing and locus repositioning linked to regulation of virulence genes in Plasmodium falciparum. Cell 2005; 121:13-24. 